\title{
Analysis of the Association of Administration of various glucocorticoids with development of acute pancreatitis using US Food and Drug Administration adverse event reporting system (FAERS)
}

Daisuke Nango ${ }^{1,3^{*}}$, Yukifumi Hirose ${ }^{1}$, Makoto Goto ${ }^{2}$ and Hirotoshi Echizen ${ }^{3}$

\begin{abstract}
Background: There have been debates about the association between the administration of glucocorticoids and the development of acute pancreatitis, since many anecdotal cases of this adverse event were affected either by concomitant diseases (such as systemic lupus erythematosus, SLE) that may develop acute pancreatitis without glucocorticoid treatment or by co-administered drugs with high risk for the event. The aim of the present study was to explore whether disproportionally elevated signals of developing acute pancreatitis may be detected in patients receiving glucocorticoids as compared those receiving other drugs.

Methods: We retrieved spontaneously reported cases of acute pancreatitis and clinically related adverse events (target events) from the US Food and Drug Administration Adverse Event Reporting System (FAERS) using 18 preferred terms (PTs). Target drugs studied were cortisol, cortisone, prednisolone, methylprednisolone, triamcinolone, dexamethasone, and betamethasone. After cleaning the data, we calculated reporting odds ratios (RORs) and 95\% confidence intervals (Cls) of acute pancreatitis in patients who received one of the glucocorticoids. RORs were calculated for each glucocorticoid using all reported cases irrespective of reporters' judgement about the contribution of the target drugs to events [i.e., primary suspected medication (PS), secondary suspected medication (SS), concomitant medication (C) and interacting (I)] and using cases with higher certainty of contribution (PS and SS), separately. When the lower limit of 95\% Cl of a ROR signal exceeded 1.0, the signal was considered statistically significant.

(Continued on next page)
\end{abstract}

\footnotetext{
* Correspondence: d.nango@gmail.com

'Departments of Pharmacy, Shin-Yurigaoka General Hospital, 255

Furusawa-tsuko, Asao-ku, Kawasaki, Kanagawa 215-0026, Japan

${ }^{3}$ Department of Pharmacotherapy, Meiji Pharmaceutical University, 2-522-1

Noshio, Kiyose, Tokyo 204-8588, Japan

Full list of author information is available at the end of the article
}

(c) The Author(s). 2019 Open Access This article is distributed under the terms of the Creative Commons Attribution 4.0 International License (http://creativecommons.org/licenses/by/4.0/), which permits unrestricted use, distribution, and reproduction in any medium, provided you give appropriate credit to the original author(s) and the source, provide a link to the Creative Commons license, and indicate if changes were made. The Creative Commons Public Domain Dedication waiver (http://creativecommons.org/publicdomain/zero/1.0/) applies to the data made available in this article, unless otherwise stated. 
(Continued from previous page)

Results: The RORs (95\% Cls) calculated using all reported cases (PS, SS, C, and I) for cortisol (1.68; 1.43-1.98), prednisolone (1.33; 1.19-1.47), methylprednisolone $(1.77 ; 1.55-2.02)$ were significant, whereas those for other target drugs were insignificant. Using the cases in which target drugs were considered to contribute the events with higher certainty (PS or SS), RORs for prednisolone (1.31; 1.10-1.55), methylprednisolone (1.62; 1.30-2.01), and dexamethasone (1.27; 1.10-1.47) were considered significant, whereas those for others were insignificant. Regarding the performance of PTs for detecting signals (RORs) associated with acute pancreatitis from FAERS database, "pancreatitis acute" gave RORs with higher significance than others, whereas more specific PTs, "haemorrhagic necrotic pancreatitis", "ischaemic pancreatitis", "pancreatic necrosis" and "pancreatitis necrotising", gave RORs with greater magnitude.

Conclusion: The present study demonstrated that the overrepresentation of signals for acute pancreatitis may be detected for prednisolone, methylprednisolone, and some others in the FAERS database.

(372 words)

Keywords: FAERS, Glucocorticoids, Acute pancreatitis, Reported odds ratio (ROR)

\section{Introduction}

A large number of cases that developed acute pancreatitis during treatment with glucocorticoids have been reported [1-14]. However, the causal relationship between the two remains controversial, since diseases with an indication for glucocorticoid therapy either as anti-inflammatory agents or antiemetics may have increased risk of developing acute pancreatitis. For instance, patients with systemic lupus erythematosus (SLE) may develop acute pancreatitis as a complication of systemic vasculitis [15], and patients with malignant neoplasia may concomitantly receive glucocorticoids as antiemetics and antineoplastic agents that may have high risk for acute pancreatitis (such as L-asparaginase and fluorouracil antineoplastics) [16]. Recently, the Ministry of Labour Health and Welfare has issued manuals of various severe adverse drug reactions (ADRs) for health professionals. As for the risk factors of acute pancreatitis, the authors described that there are many negative opinions on the causal relationship between the administration of glucocorticoid and development of acute pancreatitis [17]. Since severe acute pancreatitis could be fatal [18, 19], a rechallenge test is rarely undertaken for suspected cases of glucocorticoid-induced acute pancreatitis.

Analysis of drug-induced adverse events that have been archived in spontaneously reporting adverse drug reaction databases may be useful for detecting signals of specific adverse drug reactions in excessive frequencies over other adverse reactions [20-22]. Among the spontaneous reporting systems of adverse drug reactions operated by regulatory authorities of different countries, the US Food and Drug Administration Adverse Event Reporting System (FAERS) [23] is one of the biggest databases currently accessible to the public. In the present study, we aimed to study whether excessive signals of developing acute pancreatitis and its associated clinical conditions (including necrotizing and haemorrhagic pancreatitis) may be detected in patients receiving commonly prescribed glucocorticoids.

\section{Methods}

We retrieved relevant datasets from the FAERS database compiled from the first quarter of 1997 to the first quarter of 2017. According to the Medical Dictionary for Regulatory Activities (MedDRA) version 20.1 [24], we employed 18 preferred terms (PTs) for collecting relevant cases associated with "acute pancreatitis [Standardized MedDRA Queries (SMQ): 20000022]" and its closely related clinical conditions. The SMQ codes for the corresponding PTs are shown in Table 1. According to the FDA recommendations, we removed duplicated data and irrelevant data from the retrieved data. In FAERS data, drugs may be documented under non-proprietary (generic) names, brand names, or their abbreviations. As a result, a drug may be filed under different synonymous names. In addition, an identical pharmaceutical molecule may be filed under different chemical names depending on different pharmaceutical products (such as cortisol and hydrocortisone). Furthermore, a glucocorticoid molecule may be used either as the free base or various salt forms in the products. For instance, hydrocortisone is formulated as free base as well as sodium succinate or sodium phosphate salts in different products. The same also applies to other glucocorticoids (including dexamethasone, triamcinolone, prednisolone, betamethasone, and methylprednisolone). As a result, concomitantly administered drugs in different cases under different synonymous names had to be unified into one of the typical drug names by text-mining approach. In the present study, multi-ingredient medications containing glucocorticoids and other ingredients (such as a combination formula of betamethasone and $d$-chlorpheniramine maleate) were excluded from analysis.

For pharmacovigilance analysis, several methods have been developed to detect overrepresented signals of specific adverse drug reactions for an individual drug [20-22]. In the present study, we employed reporting odds ratio (ROR) $[20,22]$. RORs of acute pancreatitis in patients receiving 
Table 1 Preferred terms (PTs) related to acute pancreatitis (SMQ;20000022)

\begin{tabular}{ll}
\hline Code & PT \\
\hline 10033625 & Pancreatic haemorrhage \\
10033635 & Pancreatic pseudocyst \\
10033636 & Pancreatic pseudocyst drainage \\
10033645 & Pancreatitis \\
10033647 & Pancreatitis acute \\
10033650 & Pancreatitis haemorrhagic \\
10033654 & Pancreatitis necrotising \\
10033657 & Pancreatitis relapsing \\
10048984 & Pancreatic abscess \\
10052400 & Oedematous pancreatitis \\
10056277 & Pancreatorenal syndrome \\
10056975 & Pancreatic phlegmon \\
10056976 & Hereditary pancreatitis \\
10058096 & Pancreatic necrosis \\
10059029 & Cullen's sign \\
10066127 & Ischaemic pancreatitis \\
10075426 & Grey Turner's sign \\
10076058 & Haemorrhagic necrotic pancreatitis \\
\hline
\end{tabular}

various glucocorticoids were calculated according to the equation of Van Puijenbroek EP et al. [22]. Specifically, ROR was calculated as $\frac{a / b}{c / d}$; where "a" is the number of patients developing a target event (acute pancreatitis) when they received a target drug (glucocorticoid), "b" is the number of patients developing non-target adverse events, "c" is the number of patients developing the target event when they received non-target drugs, and " $\mathrm{d}$ " is the number of patients developing non-target adverse events when they received non-target drugs. In addition, 95\% confidence intervals $(\mathrm{CI})$ for the respective RORs were calculated by the following equation: $\exp \left[\ln (R O R) \pm 1.96 \sqrt{\frac{1}{a}+\frac{1}{b}+\frac{1}{c}+\frac{1}{d}}\right]$. When the lower limit of the $95 \%$ confidence interval (CI) for a ROR was greater than 1.0, the signal was considered statistically significant. We calculated $p$-values of RORs with Fisher's exact test. A p-value of $<0.05$ was considered significant.

FAERS data contain not only information about all concomitantly administered drugs in patients who were reported to develop adverse reactions but also the reporters' judgement about the contribution of each of the co-administered drugs to the adverse reaction. The reporters' judgement about the certainty of the contribution of each drug to the corresponding adverse event was graded into four levels [primary suspected medication (PS), secondary suspected medication (SS), concomitant medication (C) and interacting (I)]. In the present study, we calculated RORs using all data irrespective of the subjective judgement about certainty (overall data) as well as RORs using only those data with higher levels of certainty (classified as PS and SS).

We further analysed the data to identify the PT that most effectively detects cases of acute pancreatitis. For this purpose, we used a volcano plot. Specifically, we plotted the negative common logarithm (to the base 10) of statistical significance ( $p$ values) on the $y$-axis and the normal logarithm (to the base e) of ROR on the $\mathrm{x}$-axis for various PTs. This plot allows quick visual identification of data points displaying large magnitude signals that are also statistically significant. Statistical analyses were performed with JMP ${ }^{\bullet}$ Pro ver. 13 (SAS Institute Inc. NC, USA).

\section{Results}

We listed all preferred terms (PTs) that were employed in the present study for identifying patients who developed acute pancreatitis (SMQ) and its related clinical conditions in Table 1. We retrieved a total of 10,413,882 adverse events from the FAERS database. After data cleaning, $8,437,343$ cases were subject to analysis. We identified $16,431,3580,84,411,11,363,50,242,23,496$, and 3825 cases who developed any ADRs while receiving cortisol, cortisone, dexamethasone, triamcinolone, prednisolone, methylprednisolone, and betamethasone, respectively. These numbers corresponded to $0.19,0.04,1.00,0.13$, $0.60,0.28$, and $0.05 \%$, respectively, of the total cases of developing any ADRs that were used for analysis in the present study (i.e., 8,437,343 cases). A total of 44,893 cases were identified as developing acute pancreatitis (SMQ) while receiving any drugs including glucocorticoids. As shown in Table 2, 146, 22, 387, 50, 353, 220, and 24 cases of patients were reported developing acute pancreatitis (SMQ) while receiving cortisol, cortisone, dexamethasone, triamcinolone, prednisolone, methylprednisolone, and betamethasone, respectively. As a result, the numbers of these cases corresponded to $0.32,0.05,0.86,0.11,0.79$, 0.49 , and $0.05 \%$, of the total cases of developing of acute pancreatitis (SMQ) (i.e., 44,893 cases), respectively.

The results of analysis showed that RORs calculated for cortisol, prednisolone, and methylprednisolone were significantly $(p<0.01)$ elevated when using data including all levels of certainty (Table 2), whereas the signal of dexamethasone was significantly less than the unity. In contrast, when analysis was performed using only the data with higher certainty for the association of glucocorticoids with adverse events, RORs for prednisolone, methylprednisolone and dexamethasone were considered significantly elevated $(p<0.01)$. We considered that the signal for betamethasone was insignificant. The $p$-value of the ROR calculated with Fisher' exact test was slightly greater than 0.05 , although the lower limit of the 
Table 2 The numbers of cases of patients who developed acute pancreatitis (SMQ;20000022) during treatment with glucocorticoids and RORs for the respective glucocorticoids

\begin{tabular}{|c|c|c|c|c|c|}
\hline & Total & Case & RR (\%) & ROR (95\%Cl) & $P$-value \\
\hline \multicolumn{6}{|l|}{$\overline{\mathrm{ALL}}(\mathrm{PS}+\mathrm{SS}+\mathrm{C}+\mathrm{I})$} \\
\hline Cortisol & 16,431 & 146 & 0.89 & $1.68(1.43-1.98)$ & $<0.001$ \\
\hline Cortisone & 3580 & 22 & 0.61 & $1.16(0.76-1.75)$ & 0.49 \\
\hline Prednisolone & 50,242 & 353 & 0.70 & $1.33(1.19-1.47)$ & $<0.001$ \\
\hline Methylprednisolone & 23,496 & 220 & 0.94 & $1.77(1.55-2.02)$ & $<0.001$ \\
\hline Triamcinolone & 11,363 & 50 & 0.44 & $0.83(0.63-1.09)$ & 0.20 \\
\hline Dexamethasone & 84,411 & 387 & 0.46 & $0.86(0.78-0.95)$ & $<0.01$ \\
\hline Betamethasone & 3825 & 24 & 0.63 & $1.18(0.79-1.76)$ & 0.38 \\
\hline \multicolumn{6}{|l|}{$P S+S S$} \\
\hline Cortisol & 2865 & 16 & 0.56 & $1.05(0.65-1.71)$ & 0.80 \\
\hline Cortisone & 640 & 2 & 0.31 & $0.59(0.16-2.14)$ & 0.78 \\
\hline Prednisolone & 19,134 & 133 & 0.70 & $1.31(1.10-1.55)$ & $<0.01$ \\
\hline Methylprednisolone & 9564 & 82 & 0.86 & $1.62(1.30-2.01)$ & $<0.001$ \\
\hline Triamcinolone & 4749 & 4 & 0.08 & $0.16(0.06-0.41)$ & $<0.001$ \\
\hline Dexamethasone & 28,140 & 190 & 0.68 & $1.27(1.10-1.47)$ & $<0.01$ \\
\hline Betamethasone & 1121 & 11 & 0.98 & $1.85(1.03-3.33)$ & 0.058 \\
\hline
\end{tabular}

Case; the number of reported cases with patients who developed acute pancreatitis during treatment with each glucocorticoid (target drug), Total; number of reported cases for the corresponding glucocorticosteroids, $R R$ reporting ratio (case/total $\times 100), R O R$ reporting odds ratio, $C l$ confidence interval, $P S$ primarily suspected drug, SS secondarily suspected drug, $C$ concomitant drug, I interacting drug

95\% confidence interval was greater than 1.0 (Table 2). The signal for triamcinolone was significantly less than the unity. In addition, there was an inconsistency about the RORs of dexamethasone that were calculated with use of ALL data and PS + SS data.

In Table 3, we showed the results of disproportionality analyses performed with use of various PTs for the three glucocorticoids (i.e., prednisolone, methylprednisolone, and dexamethasone) which showed significantly overrepresented signals for acute pancreatitis (SMQ). The RORs calculated for the combinations of prednisolone or methylprednisolone and two PTs (pancreatitis acute and pancreatitis necrotizing) were significantly $(p<0.01)$ elevated irrespective of the reporters' certainty about the causality (i.e., ALL and PS + SS) (Table 3). In contrast, the results of the analyses for other three PTs (pancreatic necrosis, ischaemic pancreatitis and haemorrhagic necrotic pancreatitis) were inconclusive due mainly to a scarcity of samples.

We drew volcano plots for three glucocorticoids that showed statistically significant overrepresenting signals for acute pancreatitis (SMQ:20000022) when the analysis was conducted using data of all levels of reporters' certainty about causality (Fig. 1) and when the analysis was conducted using data of higher certainty (Fig. 2). The plots showed that the PT of "pancreatitis acute" had the highest levels of statistical significance as compared other PTs, although the magnitude of the signal was less impressive than more specific PTs, irrespective of the reporters' certainty about the causality between ADRs and administration of glucocorticoids (Figs. 1 and 2). In contrast, more specific PTs including haemorrhagic necrotic pancreatitis and ischaemic pancreatitis had greater changes in ROR signal, but their statistical significance was inferior to the PT of pancreatitis acute. Datasets for pancreatic pseudocyst drainage (PT; 10033636), pancreatorenal syndrome (PT; 10056277), pancreatic phlegmon (PT; 10056975), hereditary pancreatitis (PT; 10056976), Cullen's sign (PT; 10059029), Grey Turner's sign (PT; 10075426) were not plotted, since no cases were collected from the database.

\section{Discussion}

To our knowledge, this is the first study to investigate the signals of developing acute pancreatitis during treatment with commonly used glucocorticoids, using a large spontaneous reporting system of adverse drug reactions, FAERS. We found significant overrepresentation of signals for acute pancreatitis (SMQ:20000022) over other adverse reactions for prednisolone, methylprednisolone. These findings were observed not only from the analysis using all data irrespective of reporters' subjective judgment about the certainty for the contribution of glucocorticoids to acute pancreatitis, but also from the analysis using data judged by reporters as having higher levels of certainty (Table 2). In addition, we revealed that "pancreatitis acute" would be the best PT over others for detecting elevated signals associated with acute pancreatitis and related clinical conditions in the spontaneous reporting system, 
Table 3 The numbers of cases who were reported as developing various PTs while receiving each of the glucocorticoids and RORs for the respective combinations of PTs and drugs

\begin{tabular}{|c|c|c|c|c|c|c|}
\hline \multirow[t]{2}{*}{$\overline{\text { Drug }}$} & \multicolumn{2}{|c|}{ Case } & \multicolumn{4}{|l|}{ ROR $(95 \% \mathrm{Cl}), P$ value } \\
\hline & $\overline{\mathrm{ALL}}$ & $P S+S S$ & $\overline{\mathrm{ALL}}$ & & $P S+S S$ & \\
\hline \multicolumn{7}{|c|}{ Pancreatitis acute (PT; 10033647) } \\
\hline Prednisolone & 159 & 64 & $2.41(2.06-2.82)$ & $<0.001$ & $2.53(1.98-3.23)$ & $<0.001$ \\
\hline Methylprednisolone & 103 & 52 & $3.33(2.74-4.05)$ & $<0.001$ & $4.13(3.14-5.42)$ & $<0.001$ \\
\hline Dexamethasone & 133 & 76 & $1.19(1.00-1.41)$ & 0.051 & $2.04(1.63-2.56)$ & $<0.001$ \\
\hline \multicolumn{7}{|c|}{ Pancreatitis necrotizing (PT; 10033654) } \\
\hline Prednisolone & 13 & 6 & $2.02(1.18-3.46)$ & 0.017 & $2.44(1.12-5.33)$ & 0.040 \\
\hline Methylprednisolone & 7 & 5 & $2.32(1.12-4.80)$ & 0.035 & $4.07(1.73-9.54)$ & 0.009 \\
\hline Dexamethasone & 19 & 15 & $1.76(1.12-2.76)$ & 0.021 & $4.18(2.52-6.91)$ & $<0.001$ \\
\hline \multicolumn{7}{|c|}{ Pancreatic necrosis (PT; 10058096) } \\
\hline Prednisolone & 9 & 5 & $5.99(3.12-11.51)$ & $<0.001$ & $8.63(3.66-20.36)$ & $<0.001$ \\
\hline Methylprednisolone & 8 & 5 & $11.37(5.70-22.68)$ & $<0.001$ & $17.29(7.33-40.80)$ & $<0.001$ \\
\hline Dexamethasone & 2 & 1 & $0.77(0.21-2.81)$ & 1.000 & $1.15(0.20-6.55)$ & 0.58 \\
\hline \multicolumn{7}{|c|}{ Ischaemic pancreatitis (PT; 10066127) } \\
\hline Prednisolone & 0 & 0 & NS & NS & NS & NS \\
\hline Methylprednisolone & 0 & 0 & NS & NS & NS & NS \\
\hline Dexamethasone & 2 & 2 & $98.96(17.47-560.60)$ & 0.001 & $298.86(52.75-1692.06)$ & $<0.001$ \\
\hline \multicolumn{7}{|c|}{ Haemorrhagic necrotic pancreatitis (PT; 10076058) } \\
\hline Prednisolone & 4 & 4 & $60.71(20.42-180.51)$ & $<0.001$ & $160.02(53.82-475.83)$ & $<0.001$ \\
\hline Methylprednisolone & 4 & 4 & $130.24(43.80-387.27)$ & $<0.001$ & 320.57 (107.80-953.33) & $<0.001$ \\
\hline Dexamethasone & 0 & 0 & NS & NS & NS & NS \\
\hline
\end{tabular}

Case; numbers of cases reported as developing the respective PTs during the administration of each glucocorticoid (target drug), ROR Reporting Odds Ratio, PS Primary suspect drug, SS Secondary suspect drug, C Concomitant drug, I Interacting drug, All; PS + SS + SC + I. RORs were calculated using data to which reporters had higher certainty about causality (PS + SS) and those included all levels of reporters' certainty (ALL), separately, NS not significant

because volcano plots indicated that this PT showed by far higher significance based on $p$-value, albeit lower magnitude of changes in signal based on ROR, than more specific PTs including "haemorrhagic necrotic pancreatitis" and "ischaemic pancreatitis" (Figs. 1 and 2).

A diagnosis of drug-induced acute pancreatitis is often difficult to establish. Since acute pancreatitis is a rare and severe clinical condition with high mortality $[18,19]$, confirmation of a causal relationship between an assumed responsible drug and the event by rechallenge is difficult to conduct or ethically prohibited. In addition, patients who develop acute pancreatitis during treatment with a drug often have obvious risk factors for developing acute pancreatitis (such as alcoholism, systemic vasculitis due to immunological mechanism, and concomitant medications known to cause pancreatitis) other than the assumed perpetrator drug. Glucocorticoids have been claimed to be the aetiology of acute pancreatitis in patients receiving the drugs for the treatment of autoimmune diseases such as SLE. However, approximately $8 \%$ of patients with SLE develop acute pancreatitis irrespective of the administration of glucocorticoids [15]. Glucocorticoids were also implicated as the aetiology of acute pancreatitis in cancer patients who received glucocorticoids as antiemetic agent during anticancer chemotherapy. However, those patients are often given antineoplastic agents concomitantly, which are known to cause acute pancreatitis per se [16].

Besides anecdotal case reports of acute pancreatitis in patients receiving glucocorticoids, two lines of evidence may support the relationship between the administration of glucocorticoids and development of acute pancreatitis. Recently, we reported a patient with a diagnosis of autoimmune hepatitis $(\mathrm{AIH})$ who developed acute pancreatitis immediately after administration of methylprednisolone [25] for the treatment of AIH. Since AIH has never been reported to be complicated with acute pancreatitis in the literature and the patient had no other possible causes of the event, we considered that there was a causal relationship between the administration of methylprednisolone and acute pancreatitis. Another approach to search for a signal of overrepresentation of acute pancreatitis during the administration of glucocorticoids over other drugs is using a large spontaneous reporting system of adverse drug reactions, such as FAERS. In the present study, we observed that RORs of acute pancreatitis for three glucocorticoids; prednisolone, methylprednisolone and 


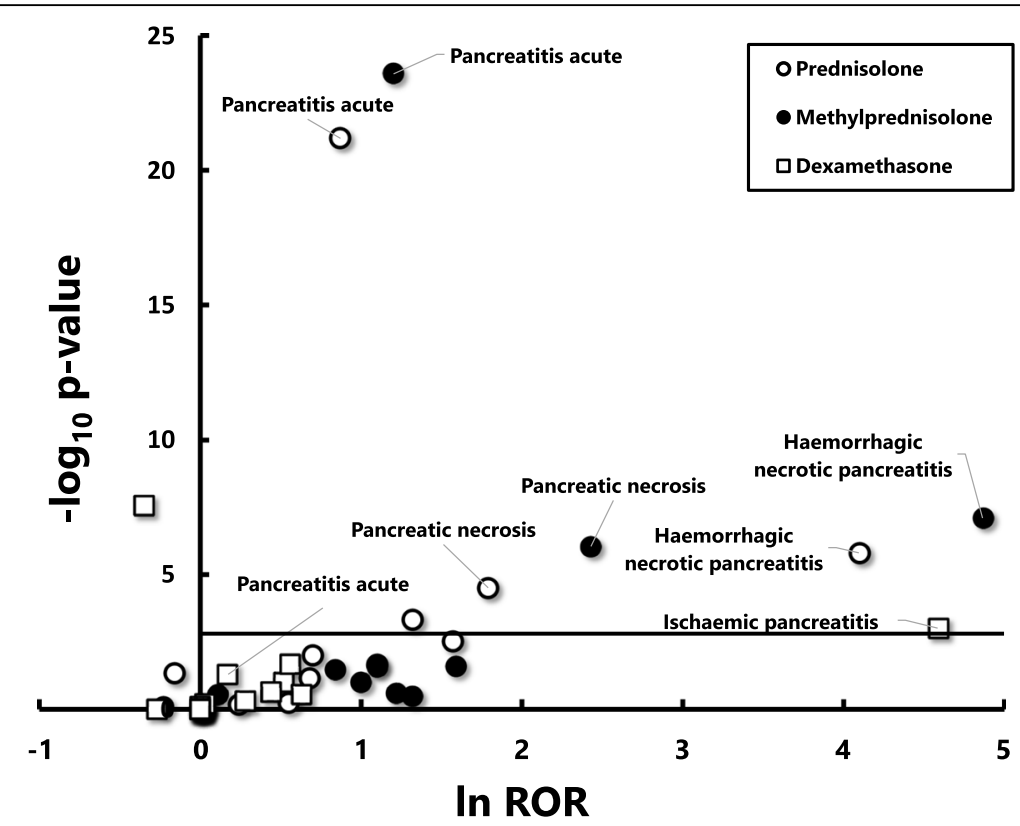

Fig. 1 A volcano plot for visualizing statistical significance (p-values) and the magnitude of alarm signals (reporting odds ratios; RORs) for 18 PTs that were used for detecting the development of acute pancreatitis (SMQ) during the administration of prednisolone, methylprednisolone and dexamethasone. RORs were calculated using the reported data including all levels of reporters' certain about the causalityNegative common logarithm (to the base 10) of $p$-values $(-\log 10$ P) are plotted on the $y$-axis and natural logarithm (to the base e) of RORs (In ROR) are plotted on $x$-axis. The horizontal line represents the threshold of significance $(p=0.05)$ corrected for multiple comparisons by Bonferroni's method $(p=0.00093)$

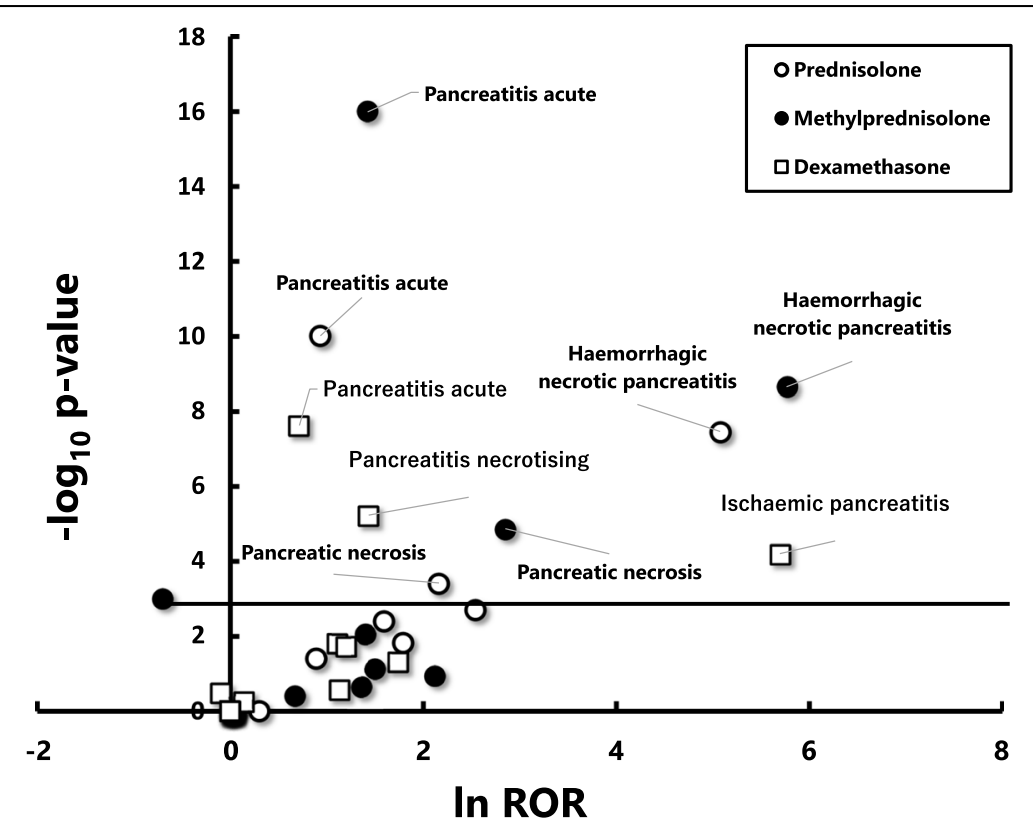

Fig. 2 A volcano plot for visualizing statistical significance ( $p$-values) and the magnitude of alarm signals (reporting odds ratios; RORs) for 18 PTs. RORs were calculated using the reported data to which reporters had higher certainly about causality (primary and secondary suspected) for prednisolone, methylprednisolone and dexamethasoneNegative common logarithm (to the base 10) of p-values (-log10 P) are plotted on the $y$ axis and natural logarithm (to the base e) of RORs (In ROR) are plotted on x-axis. The horizontal line represents the threshold of significance ( $p=$ 0.05) corrected for multiple comparisons by Bonferroni's method $(p=0.00093)$ 
dexamethasone, were significantly $(p<0.05)$ overrepresented with higher certainty (Table 2). Collectively, the present study may further support a causal relationship between the administration of glucocorticoids and the development of acute pancreatitis.

Badalov et al. [26] reported an evidence-based review on drug-induced acute pancreatitis. They reviewed literature data of drugs that were suspected to be associated with the aetiology of acute pancreatitis based on the weight of evidence for each agent and pattern in presentation. They classified drugs into four classes according to the certainty of the causal relationship. Class I drugs include medications in which at least one case report described a recurrence of acute pancreatitis with a rechallenge. No glucocorticoids were included in this class. Dexamethasone and prednisolone were classified into Class II, and other glucocorticoids were unclassified due to a lack of relevant data. Sadr-Azodi et al. [27] undertook a population-based nested case-control study in Swedish population and found that oral glucocorticoid use was associated with an increased risk of developing acute pancreatitis (odds ratio, 1.53; 95\% CI, 1.27-1.84) compared with nonusers. Unfortunately, they did not analyse the contribution of individual glucocorticoids to the overall risk of acute pancreatitis separately. Collectively, these previous studies are in good agreement with the present study.

The present study provides an auxiliary finding about the selection of PTs for effective detection of a target adverse event. The volcano plots allowed visualization of the statistical significance and magnitude of signals for 18 PTs related to acute pancreatitis for prednisolone, methylprednisolone and dexamethasone simultaneously (Figs. 1, 2). The plots demonstrated that the PT, "pancreatitis acute", detected signals (RORs) having by far the greatest statistical significance, albeit the less impressive magnitude in signal compared with others. In contrast, more specific PTs including haemorrhagic necrotic pancreatitis and ischaemic pancreatitis detected signals (RORs) with greater magnitude, but less significance, than the PT of "pancreatitis acute". While this finding does not contradict our intuitive understanding, whether similar finding may be observed for the analysis of other drug-induced adverse reactions remains to be confirmed.

Spontaneous reporting systems for suspected adverse drug reactions are considered the cornerstone of pharmacovigilance, since they may detect potential alarm signals related to drug use. However, caution should be exercised when utilizing spontaneous reporting systems, since there are inherent limitations and obstacles (e.g., under-reporting, selective reporting, lack of information about total drug consumption, and many others) $[28,29]$. As a result, the results of disproportionality analyses using spontaneously reporting ADR databases should be only considered as exploratory in a context of signal detection and it does not allow quantification of the true risk [28, 29] These limitations and obstacles may also exist in the present study. For example, clinical data of reported cases are often incomplete for detailed analysis in the present study. We were unable to search for clinical risk factors of acute pancreatitis including alcohol intake [30], the presence of cholelithiasis [31], hyperlipidaemia [32], obesity [33], and information of latency of the event. Also, information about concomitant medications such as immunosuppressive drugs (such as azathioprine [34]) that are classified as high risk (Class I) drugs of acute pancreatitis is often unavailable in the present study. Since these drugs may have been co-administered with glucocorticoids, further studies are required in the future.

In addition, there were some inconsistency among the results of the disproportionality analyses. As for dexamethasone, the ROR for acute pancreatitis (SMQ) that was calculated using the data including all levels of reporters' certainly about the causality (i.e., ALL) was significantly less than the unity, but that calculated using the data for which reporters had higher certainty (i.e., PS + SS) was significantly greater than the unity (Table 2 ). We cannot offer any definitive explanations about this finding. Nevertheless, we recognized that the reporting ratio of acute pancreatitis (SMQ) for dexamethasone listed in Table 2 for the PS + SS data $(0.68 \%)$ was approximately $50 \%$ greater than that for the ALL data $(0.46 \%)$. This finding suggests that dexamethasone may have been reported more likely as primary suspected (PS) or secondary suspected (PS) drugs than as concomitant drug (C) or interacting drug (I) for concomitantly observed acute pancreatitis. Nevertheless, we cannot categorically negate a possibility that there may be a selective reporting bias of reporters due to their preconceived notion about a causality between the administration of steroids and development of pancreatitis.

\section{Conclusions}

Using pharmacoepidemiologic approach, we demonstrated that there is increased risk of developing acute pancreatitis in patients receiving at least one of three glucocorticoids. While the present study does not prove the causal relationship between the administration of glucocorticoids and the development of acute pancreatitis, it may advance our understanding of this clinical issue.

\section{Abbreviations \\ Cl: Confidence interval; FAERS: The Food and Drug Administration Adverse Event Reporting System; PT: Preferred Term; ROR: Reporting odds ratios; SLE: Systemic lupus erythematosus; SMQ: Standardized MedDRA Queries; SRS: Spontaneous reporting systems}

\section{Acknowledgements}

Not applicable.

\section{Funding}

The authors received no financial support for the research, authorship, and/ or publication of the present article. 


\section{Availability of data and materials}

The dataset analysed in the present study are open to the public

\section{Authors' contributions}

DN and MG performed data curation. DN conducted data analysis. DN and HE planned the study protocol and wrote the manuscript. MG and YH helped drafting the manuscript. All authors read and approved the final manuscript.

\section{Ethics approval and consent to participate}

Ethical approval was not sought because the study involved only analysis of data obtained from a public database. Informed consent to participate: not applicable.

\section{Consent for publication}

Not applicable.

\section{Competing interests}

The authors declare that they have no competing interest.

\section{Publisher's Note}

Springer Nature remains neutral with regard to jurisdictional claims in published maps and institutional affiliations.

\section{Author details}

1Departments of Pharmacy, Shin-Yurigaoka General Hospital, 255 Furusawa-tsuko, Asao-ku, Kawasaki, Kanagawa 215-0026, Japan. ${ }^{2}$ Kyoto Constella Technologies Co., Ltd., 4th Floor, Kyozome Kaikan, 481 Tourouyama-cho, Nakagyo-ku, Kyoto 604-8225, Japan. ${ }^{3}$ Department of Pharmacotherapy, Meiji Pharmaceutical University, 2-522-1 Noshio, Kiyose, Tokyo 204-8588, Japan.

Received: 25 October 2018 Accepted: 23 January 2019

Published online: 28 February 2019

\section{References}

1. Jones MR, Hall OM, Kaye AM, Kaye AD. Drug-induced acute pancreatitis: a review. Ochsner J. 2015:15:45-51.

2. Okumura H, Miyahara K, Ito K, Koshimura O, Hashimoto K. Review of literature and a case report of steroid pancreatitis. Naika. 1969;23:915-20 (In Japanese).

3. Jain R, Ramanan SV. latrogenic pancreatitis. A fatal complication in the induction therapy for acute lymphocytic leukemia. Arch Intern Med. 1978;138:1726

4. Hamed I, Lindeman RD, Czerwinski AW. Case report: Acute pancreatiti following corticosteroid and azathioprine therapy. Am J Med Sci. 1978;276:211-9.

5. Baron M, Brisson ML. Pancreatitis in systemic lupus erythematosus. Arthritis Rheum. 1982;25:1006-9.

6. Ossi E, Fiocco U, Belloni M, Ongaro G, Rubaltelli L, Ruffatti A, et al. Therapy of acute pancreatitis in systemic lupus erythematosus with plasmapheresis and corticosteroids. Clin Exp Rheumatol. 1983;1:345-7.

7. Felig DM, Topazian M. Corticosteroid-induced pancreatitis. Ann Intern Med. 1996:124:1016

8. Yoshizawa Y, Ogasa S, Izaki S, Kitamura K. Corticosteroid-induced pancreatitis in patients with autoimmune bullous disease: case report and prospective study. Dermatology. 1999;198:304-6.

9. Khanna S, Kumar A. Acute pancreatitis due to hydrocortisone in a patient with ulcerative colitis. J Gastroenterol Hepatol 2003;18:1110-1.

10. Tsuruoka K, Sekiya S, Yokoyama T, Koitabashi K, Shimazaki M, Sakurada T, et al. MPO-ANCA related vasculitis complicating mucinous cystadenoma of the pancreas and severe acute pancreatitis after steroid pulse therapy: a case report. Nihon Jinzo Gakkai Shi. 2008:50:948-53 (in Japanese).

11. Nishimoto M, Koh H, Bingo M, Yoshida M, Nanno S, Hayashi $Y$, et al. Posterior reversible encephalopathy syndrome following acute pancreatitis during chemotherapy for acute monocytic leukemia. Rinsho Ketsueki. 2014:55:552-7 (in Japanese).

12. Sabre A, Guthrie MM, Maleknia R. Acute necrotising pancreatitis derived from low-dose corticosteroid use: an important reminder of clinical management. BMJ Case Reports. 2015. https://doi.org/10.1136/bcr-2015-209325.
13. Makol A, Petri M. Pancreatitis in systemic lupus erythematosus: frequency and associated factors-a review of the Hopkins lupus cohort. J Rheumatol. 2010;37:341-5

14. Yassin N, Buchler M, UhI W, Scherbaum WA. Antineutrophil cytoplasmic autoantibodies (ANCA) in acute pancreatitis. Hepato-Gastroenterology. 1992;39:533-5.

15. Hoffman BI, Katz WA. The gastrointestinal manifestations of systemic lupus erythematosus: a review of the literature. Semin Arthritis Rheum. 1980;9. 237-47.

16. Rünzi M, Layer P. Drug-associated pancreatitis: facts and fiction. Pancreas. 1996;13:100-9.

17. Manuals for ways of coping with severe adverse drug reactions: acute pancreatitis (drug-induced). The Ministry of Labour, Health, and Welfare. 2009. https://www.mhlw.go.jp/topics/2006/11/dl/tp1122-1g13.pdf. Accessed 17 Dec 2018.

18. Fagenholz PJ, Castillo CF, Harris NS, Pelletier AJ, Camargo CA Jr. Increasing United States hospital admissions for acute pancreatitis, 1988-2003. Ann Epidemiol. 2007;17:491-7.

19. Anderson V, Sonne J, Anderson M. Spontaneous reports on drug-induced pancreatitis in Denmark from 1968 to 1999. Eur J Clin Pharmacol. 2001:57:517-21.

20. Rothman KJ, Lanes S, Sacks ST. The reporting odds ratio and its advantages over the proportional reporting ratio. Pharmacoepidemiol Drug Saf. 2004;13: 519-23.

21. Evans SJ, Waller PC, Davis S. Use of proportional reporting ratios (PRRs) for signal generation from spontaneous adverse drug reaction reports. Pharmacoepidemiol Drug Saf. 2011;10:483-6.

22. Van Puijenbroek EP, Bate A, Leufkens HG, Lindquist M, Orre R, Egberts AC. A comparison of measures of disproportionality for signal detection in spontaneous reporting systems for adverse drug reactions. Pharmacoepidemiol Drug Saf. 2002;11:3-10.

23. FAERS database US FDA. 2018. http://www.fda.gov/default.htm. Accessed 30 April, 2017.

24. MedDRA. 2017. https://www meddra.org/. Accessed 30 September, 2017.

25. Nango D, Nakashima $H$, Hirose $Y$, Shiina M, Echizen $H$. Causal relationship between acute pancreatitis and methylprednisolone pulse therapy for fulminant autoimmune hepatitis: a case report and review of literature. J Pharm Health Care Sci. 2018:4:14.

26. Badalov N, Baradarian R, Iswara K, Li J, Steinberg W, Tenner S. Drug-induced acute pancreatitis: an evidence-based review. Clin Gastroenterol Hepatol. 2007;5:648-61.

27. Sadr-Azodi O, Mattsson F, Bexlius TS, Lindblad M, Lagergren J, Ljung R, et al. Association of oral glucocorticoid use with an increased risk of acute pancreatitis: a population-based nested case-control study. JAMA Intern Med. 2013:173:444-9.

28. Montastruc JL, Sommet A, Bagheri H, Lapeyre-Mestre M. Benefits and strengths of the disproportionality analysis for identification of adverse drug reactions in a pharmacovigilance database. Br J Clin Pharmacol. 2011;72: 905-8.

29. Palleria C, Leporini C, Chimirri S, Marrazzo G, Sacchetta S, Bruno L, et al. Limitations and obstacles of the spontaneous adverse drugs reactions reporting: two "challenging" case reports. J Pharmacol Pharmacother. 2013; 4(Suppl 1):S66-72.

30. Dufour MC, Adamson MD. The epidemiology of alcohol-induced pancreatitis. Pancreas. 2003;27:286-90.

31. Moreau JA, Zinsmeister AR, Melton LJ 3rd, DiMagno EP. Gallstone pancreatitis and the effect of cholecystectomy: a population-based cohort study. Mayo Clin Proc. 1988:63:466-73.

32. Fortson MR, Freedman SN, Webster PD 3rd. Clinical assessment of hyperlipidemic pancreatitis. Am J Gastroenterol. 1995:90:2134-9.

33. Martínez J, Sánchez-Payá J, Palazón JM, Suazo-Barahona J, Robles-Díaz G, Pérez-Mateo M. Is obesity a risk factor in acute pancreatitis? A meta-analysis. Pancreatology. 2004;4:42-8

34. Venkatesh PG, Navaneethan U. Azathioprine induced pancreatitis in a patient with co-existing autoimmune pancreatitis and hepatitis. JOP. 2011; 12:250-4 